\title{
Does Lymphatic Involvement Impact Survival of Early Stage Cervical Cancer Patients?
}

\author{
Falk Thiel Matthias W. Beckmann \\ Frauenklinik, Universitätsklinikum Erlangen, Germany
}

Lymph node metastasis is one of the most important prognostic factors for patients with cervical cancer [1]. A 5-year survival rate of $90 \%$ is reported in patients surgically treated with no evidence of lymph node metastasis. On the other hand, patients with positive pelvic or paraaortic lymph nodes have a 5-year survival of $50-60 \%$ and $20-45 \%$, respectively. However, until now, these facts are not reflected in the FIGO classification of cervical cancer which is the only one still based on clinical/diagnostic examination. Nevertheless, in daily routine nodal status is already important not only for the prognosis also for therapeutical decisions.

For this reason, Metindir and Bilir [2] performed their evaluation of 92 patients with FIGO I and II carcinoma of the cervix treated by Type II-III radical hysterectomy and bilateral pelvic and periaortic lymphadenectomy in a single institution. Patients with paraaortic lymph node involvement were excluded. For adjuvant therapy the patients with pelvic lymph node metastases, parametrial invasion, positive or close surgical margins, or lymphovascular space invasion (LVSI) with deep stromal invasion were generally considered for radiotherapy (RT) or chemoradiotherapy $(\mathrm{CT}+\mathrm{RT})$.

The main issue the authors addressed was to examine the use of the number of lymph node metastases and the ratio of positive lymph nodes to excised lymph nodes as prognostic factor for the survival of patients with early stage cervical cancer. From 92 patients, a total of 2,085 lymph nodes were removed and examined. 74 patients $(80.4 \%)$ had no involved lymph node, 5 patients $(5.4 \%)$ had one involved lymph node, and 13 patients $(14.1 \%)$ had more than one involved lymph node. In these 3 subgroups $(0 \mathrm{LN}+, 1 \mathrm{LN}+,>1 \mathrm{LN}+)$, the 5 -year survival was $89.19 \%, 100 \%$, and $76.92 \%$, respectively. These results were not statistically significant $(\mathrm{p}=0.2978)$. The authors also examined the impact of the ratio between metastatic and examined lymph nodes (LNR) on 5-year survival. They established 3 subgroups: LNR 0\%, 1-10\%, and $>10 \%$. The 5 -year survival rates were $89.1 \%, 90 \%$, and $75 \%(\mathrm{p}=0.4824)$.

The authors admit that the relatively small number of patients in the study limits the power of calculations so that no significance was reached. In patients with FIGO III-IV endometrial cancer, Chan et al. [3] have recently demonstrated a multivariate analysis that the 5-year survival is associated with an increasing lymph node ratio $(\leq 10 \%,>10-50 \%$, and $>50 \%)$, the absolute number of positive lymph nodes, and the extent of lymphadenectomy ( $\leq 20 \mathrm{LN}$ vs. $>20 \mathrm{LN})$. Metindir and Bilir concluded that the ratio (LNR) between metastatic and examined lymph nodes might also be helpful in better prognostic discrimination of patients with nodal positive cervical cancer. This has to be proven in further investigations.

Actually, there are reports from several studies demonstrating statistically significant differences in survival rates if a metastasis is present in a single lymph node (83.3-87\%) compared to multiple lymph nodes (33.1-61.7\%) [1]. It was reported that there is no difference in survival between patients with no lymph node metastasis and one lymph node metastasis $(\mathrm{p}=0.40)$. In contrast, the 5-year survival of patients with 3 or more pelvic metastases approximated the survival of patients with positive paraaortic lymph nodes ( $20.2 \%$ and $16.7 \%$, respectively). Other studies do not demonstrate a difference between one positive pelvic lymph node and multiple positive lymph nodes which may be due to the adjuvant treatment. The adjuvant treatment of cervical cancer patients with one single positive lymph node is variable among practitioners.

Lymphadenectomy in cervical cancer is important to disclose the differences in survival by presence of lymph node metastasis, location of metastasis, and perhaps the number of nodes involved or the lymph node ratio. The presence of lymph node metastasis not only gives important prognostic hints, but also helps determine the need for subsequent adjuvant therapy.

\section{KARGER}

Fax +497614520714

Information@Karger.de

www.karger.com (c) 2009 S. Karger GmbH, Freiburg

Accessible online at:

www.karger.com/onk
Prof. Dr. Matthias W. Beckmann

Frauenklinik

Universitätsklinikum Erlangen

Universitätsstraße 21-23, 91054 Erlangen, Germany

Tel.+49 9131 85-33451/2, Fax -33456

fk-direktion@uk-erlangen.de 


\section{References}

1 Gien LT, Covens AJ: Lymph node assessment in cervical cancer: Prognostic and therapeutic implications. Surg Oncol 2008 Dec 1. [Epub ahead of print]
2 Metindir J, Bilir G: Impact of the Ratio between Metastatic and Examined Lymph Nodes on Survival of Early Stage Cervical Cancer Patients. Onkologie 2009;32: DOI: 10.1159/000197361.
3 Chan JK, Kapp DS, Cheung MK, Osann K, Shin JY, Cohn D, Seid PL: The impact of the absolute number and ratio of positive lymph nodes on survival of endometrioid uterine cancer patients. $\mathrm{Br} \mathrm{J}$ Cancer 2007;97:605-611. 